\section{NTA NEWS}

\section{Tagungsband erschienen: TA im politischen System}

Das Spannungsfeld zwischen Wissenschaft und Politik steht im Mittelpunkt der Neuerscheinung, die die Beiträge zur fünften Konferenz des Netzwerks Technikfolgenabschätzung (NTA5) dokumentiert. Welche Rolle spielt TA heute in der Politik? Welchen Herausforderungen und Spannungsfeldern ist sie bei ihrer Arbeit ausgesetzt, etwa wenn es um die Beurteilung politischer und gesellschaftlicher Rahmenbedingungen neuer Technologien oder um die Einbeziehung von Bürgerinnen und Bürger geht? Welche Faktoren stehen der Umsetzung ,wissenschaftlich bester“ Lösungen durch politische Akteure im Wege?

Der Tagungsband versammelt 19 Beiträge und 8 Kurzberichte. Deren thematische Bandbreite reicht von neuen Ansätzen zur methodischen Weiterentwicklung der Technikfolgenabschätzung, über Erfahrungen im Spannungsfeld zwischen Politik und Wissenschaft bis hin zur Beschreibung bestehender Konflikte und den Facetten einer wissenschaftlichen Versachlichung der Diskussion über Technikfragen. Thematisiert werden darüber hinaus konkrete Erfahrungen im Umgang mit TA in Österreich und Belgien sowie die ethische und moralische Dimension der TA. Besonders hervorzuheben ist, dass auch die „Nachfrageseite“ der TA zu Wort kommt. „Technikfolgenabschätzung im politischen System" dokumentiert eine von Sergio Bellucci, dem Geschäftsführer von TA-Swiss, moderierte Podiumsdiskussion mit der Schweizer Nationalrätin Ruth Humbel, der Vorsitzenden des Ausschusses für Forschung, Technologie und Innovation des österreichischen Nationalrats, Ruperta Lichtenecker, und der ehemaligen Vorsitzenden des Ausschusses für Bildung, Forschung und Technikfolgenabschätzung des Bundestags, Ulla Burchardt. Die Parlamentarierinnen äußern dabei u. a. den Wunsch nach noch besseren Kommunikationsfähigkeiten der TA.

Bibliografische Angaben: Decker, M.; Bellucci, S.; Bröchler, St.; Nentwich, M.; Rey, L.; Sotoudeh, M. (Hg.): Technikfolgenabschätzung im politischen System. Zwischen Konfliktbewältigung und Techno- logiegestaltung, Berlin: edition sigma 2014 (Gesellschaft - Technik - Umwelt, Neue Folge 17)

\section{$\ll 》$}

\section{NTA goes international...}

Viele institutionelle und persönliche Mitglieder des Netzwerks TA beteiligen sich aktiv an der 2. europäischen TA-Konferenz, die im Rahmen des EU-Projekts PACITA („,Parliaments and Civil Society in TA“) Ende Februar 2015 in Berlin stattfindet. Zehn Jahre nach der ersten NTA-Konferenz kann das Netzwerk auf sechs NTA-Konferenzen und zehn Jahrestreffen zurückblicken. Die vorrangige Mission des Netzwerks ist und bleibt, eine Plattform zu sein für den Informationsaustausch zwischen deutschsprachigen Wissenschaftlerinnen und Wissenschaftlern, TA-Experten und „Praktikern“ im breit verstandenen Themenfeld TA. Gleichzeitig bringt sich das Netzwerk aber gerne in die internationalen wissenschaftlichen Debatten zu aktuellen Fragen der TA ein. Auf Initiative des NTA werden auf der PACITA-Konferenz u. a. Sessions zu den Themen „Governance of Big Data and the Role of Technology Assessment", ,Responsible Research and Innovation in Europe - First Lessons Learned“, „Horizons and Incentives of Technology Assessment“, „Varieties of Technology Governance and Opportunities for Technology Assessment" angeboten.

Alle Informationen zur Konferenz ,The Next Horizon of Technology Assessment" finden sich unter http://berlinconference.pacitaproject.eu/.

\section{$\ll »$}

\section{Save the Date!}

Das nächste Jahrestreffen des Netzwerks TA findet am 26. November 2015 um 16 Uhr in Karlsruhe statt. Dieses Treffen möchte ITAS zum Anlass nehmen, gemeinsam mit Kolleginnen und Kollegen aus dem NTA sein zwanzigjähriges Bestehen zu feiern. Am 27. November 2015 ist ein Workshop zum Thema „Institutionelle Settings in der TA“"geplant.

Michael Decker 